\title{
ELECTRON DYNAMICS OF THE ROD-PINCH DIODE IN THE CYGNUS EXPERIMENT AT LOS ALAMOS *
}

\author{
L. Yin ${ }^{\dagger}$, T. J. T. Kwan, B. G. DeVolder, C. M. Snell, K. J. Bowers, J. R. Smith, R. Carlson, \\ LANL, Los Alamos, NM 87545, USA, and M. J. Berninger, Bechtel Nevada, Los Alamos Operations
}

\begin{abstract}
In this work, two-dimensional particle-in-cell simulations are used to examine the electron physics in the rod-pinch diode, a device that can be used to produce a relatively low-energy (a few $\mathrm{MeV}$ ) radiographic electron source. It is found that with diode parameters for which the electrons' dominant dynamics are approximated well as a magnetized fluid, the diode produces an electron source with a desired small spot size as the electrons drift to and impinge on the anode tip. However, for a large cathode-toanode radius ratio, a population of electrons that consists predominantly of electrons emitted from the downstream surface of the cathode is found to propagate in the upstream direction and the diode may perform anomalously as a consequence. A method is proposed for improving the quality of the electron source by suppressing electron emission from the downstream cathode surface to reduce the presence of unmagnetized electrons.
\end{abstract}

\section{INTRODUCTION}

The Cygnus radiographic facility in operation at the Los Alamos National Laboratory [1] uses a rod-pinch diode [2] to produce a low-energy (up to $\sim 2.25 \mathrm{MeV}$ ) electron source for bremsstrahlung production. The rodpinch diode, which has been operated successfully for voltages of up to $6 \mathrm{MV}[3,4,5,6]$, consists of an annular cathode and a needle anode rod that extends through the cathode aperture. As the electrons deposit their energies on the high-atomic-number anode/converter tip, bremsstrahlung photons are produced which can be used to generate radiographs of an object.

In Cygnus experiments, two distinct types of rod-pinch diode performance are observed. For a $0.75 \mathrm{~mm}$-diameter anode rod and a $9 \mathrm{~mm}$-diameter aperture cathode (with a cathode-to-anode radius ratio of $r_{c} / r_{a}=12$ ), the experiments yield repeatable measurements of diode voltage and current. In contrast, for a $0.5 \mathrm{~mm}$-diameter anode rod and a $8 \mathrm{~mm}$-diameter aperture cathode $\left(r_{c} / r_{a}=16\right)$, the measurements are inconsistent from shot to shot and the diode behaves anomalously with a low impedance [1]; moreover, electrons are sometimes observed propagating upstream rather than pinching at the anode tip as expected from the diode design [7]. Although erratic performance of the rodpinch diode has been associated with a large $r_{c} / r_{a}$ ratio

\footnotetext{
${ }^{*}$ This work was performed under the auspices of the U.S. Department of Energy by the University of California Los Alamos National Laboratory under contract No. W-7405-Eng-36.

† lyin@lanl.gov
}

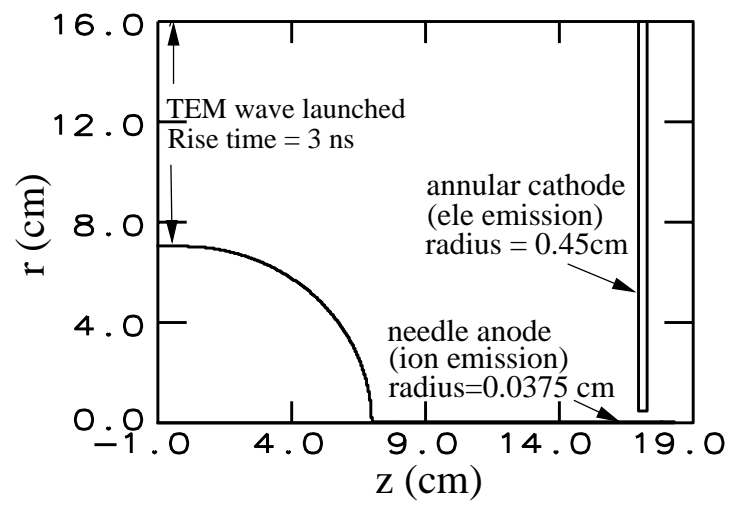

Figure 1: The configuration of the 2-D simulation.

and finite electron angular momentum [8], the exact mechanism for the anomalous behavior has not been understood.

To aid the analysis of the Cygnus experiments, the twodimensional (2-D) particle-in-cell (PIC) code MERLIN [9] is used to examine the dynamics of the rod-pinch electron source. In simulations, transverse electromagnetic (TEM) waves are launched at one simulation boundary to set up the voltage required for the electron emission and both electron and ion trajectories are followed self-consistently in the electromagnetic fields. Various diode parameters with different $r_{c} / r_{a}$ ratios are modeled to examined both the drift motion of a magnetized electron fluid and the electrons' unmagnetized dynamics. The electrons emitted from different cathode surfaces are tagged and analyzed separately in an attempt to explain the anomalous diode performance seen in Cygnus experiments and to explore a possible technique for improving the quality of the electron source for flash X-ray radiography. Simulation results using a large $r_{c} / r_{a}$ ratio show that the electrons propagating upstream and striking the anode stalk as observed in Cygnus experiments come primarily from the downstream surface of the cathode. These electrons are drawn upstream in a region where the axial electric field is downstream directed and the magnetic effects are weak (these electrons can be described as unmagnetized electrons in this region). This downstream directed axial electric field is present as a result of other electrons' motion towards the anode $\left(\mathbf{E} \simeq-\mathbf{v}_{\mathbf{e}} \times \mathbf{B}\right)$. A method for improving the rod-pinch diode performance by suppressing the electron emission from the downstream cathode surface is discussed. 

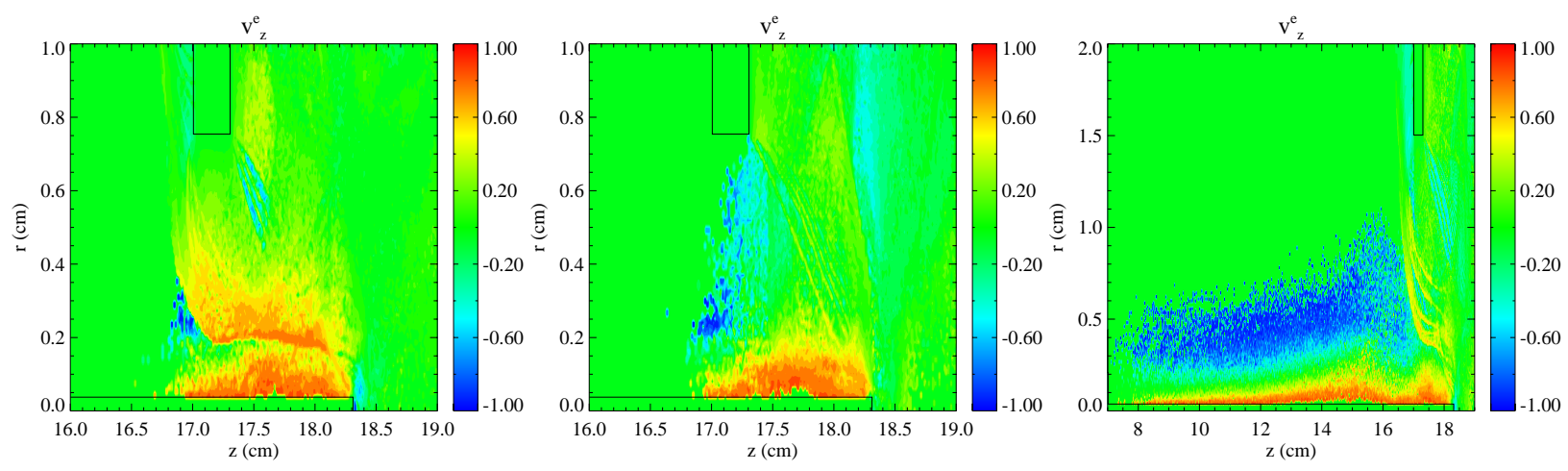

Figure 2: The electron fluid velocity $v_{z}^{e}$ from simulations with larger cathode-to-anode radius ratios, $r_{c} / r_{a}=20$ (left and middle panels) and $r_{c} / r_{a}=40$ (right panel) at $t=10 \mathrm{~ns}$. See text for details.

\section{PARTICLE-IN-CELL SIMULATIONS}

The configuration of the 2-D simulation is shown in Figure 1 , where $z$ and $r$ are the axial and radial coordinates. The spatial domain of the simulation extends $21.55 \mathrm{~cm}$ and $19.04 \mathrm{~cm}$ in the axial and radial directions, respectively, and only part of the simulation domain is displayed. This size is comparable to that of the rod-pinch diode used in the Cygnus experiment. The curved surface at the bottom left corner of this panel is the anode stalk which is connected to the anode rod centered around the $z$-axis. The tip of the anode extends through the annular cathode aperture by an axial distance of $1 \mathrm{~cm}$ measured from the downstream (right) cathode surface.

For the simulations in this work, a $0.75 \mathrm{~mm}$-diameter anode rod and a variable cathode aperture are modeled. The TEM waves are launched at the left (upstream) boundary to set up the voltage required for emitting electrons from the cathode surface with a space-charge limited emission threshold of $50 \mathrm{kV} / \mathrm{cm}$ After a 3 ns rise time, the TEM waves are applied at full amplitude and the system reaches a steady-state after $t>10 \mathrm{ns,} \mathrm{during} \mathrm{which} \mathrm{the} \mathrm{diode} \mathrm{volt-}$ age is maintained at $\simeq 2.25 \mathrm{MV}$. Hydrogen ions with realistic mass are emitted from the anode surface as the electric field exceeds a threshold of $100 \mathrm{kV} / \mathrm{cm}$ (the emitted electrons and ions have zero momenta initially). The presence of ions allows for a charge neutralization of the local electric field to occur so that the electrons can develop a drift toward the anode tip.

The diode voltage, current, and electron energy from simulations using parameters from the Cygnus experiment $\left(r_{c} / r_{a}=12\right)$ are consistent with the peak values obtained from the Cygnus experiments [1]. The fluid quantities extracted from our simulations show that the electron dynamics is well approximated as a magnetized fluid and the diode produces an electron source with the desired small spot size [10].

With larger $r_{c} / r_{a}$ ratios, however, the electrons in a rod-pinch diode exhibit unmagnetized behavior. Figure 2 shows the electron fluid velocity $v_{z}^{e}$ from simulations with $r_{c} / r_{a}=20$ (left and middle panels) and $r_{c} / r_{a}=40$ (right panel), both at $t=10 \mathrm{~ns}$. The axial velocity $v_{z}^{e}$ in the left panel is computed from all the electrons in the simulation and it indicates a main magnetized electron flow to the downstream (in red) and a small population of electrons that are moving upstream (indicated by the blue color). The electromagnetic field configuration indicates that the electrons emitted from the upstream (left) and bottom surfaces of the cathode travel through regions of relatively large fields. Consequently, these electrons become magnetized and their axial motion is dominated by a fluid drift $E_{r} \times B_{\theta}$ to the tip as they approach the anode. In contrast, the upstream propagating electrons are coming from the downstream cathode surface. Initially, they pass through a region in which a downstream directed axial electric field is present but the magnetic effects are weak. These electrons' dynamics are dominated by the electric field $E_{r}$ and $E_{z}$ only. They would undergo $E_{r} \times B_{\theta}$ drift downstream only when they eventually arrive at the region near the anode shaft.

To confirm this, the middle panel displays the electron fluid velocity computed from electrons emitted from the downstream cathode surface alone for the same run $\left(r_{c} / r_{a}=20\right)$. It is clear that after these electrons leave the cathode surface they first propagate to the upstream (and at the same time propagate downward), as indicated by the blue region, then begin to undergo $E_{r} \times B_{\theta}$ drifts to the tip along the anode shaft where the fields are strong, as seen in red. For a even larger $r_{c} / r_{a}=40$, the upstream propagating electrons can reach far back to the location of the anode stalk (at $z \sim 7 \mathrm{~cm}$ ) as seen in the right panel of Figure 2 . In this panel, the fluid velocity $v_{z}^{e}$ is computed from all the electrons. However, these upstream-propagating electrons consist predominantly of electrons emitted from the downstream cathode surface. They eventually drift to the anode tip along a large area of the anode shaft.

We propose to reduce the presence of these undesirable upstream-propagating electrons from the rod-pinch diode in Cygnus experiments by treating the downstream cathode surface via polishing or oiling to suppress the electron emission in order to improve the diode performance at large $r_{c} / r_{a}$. Two simulation runs testing this method are shown 

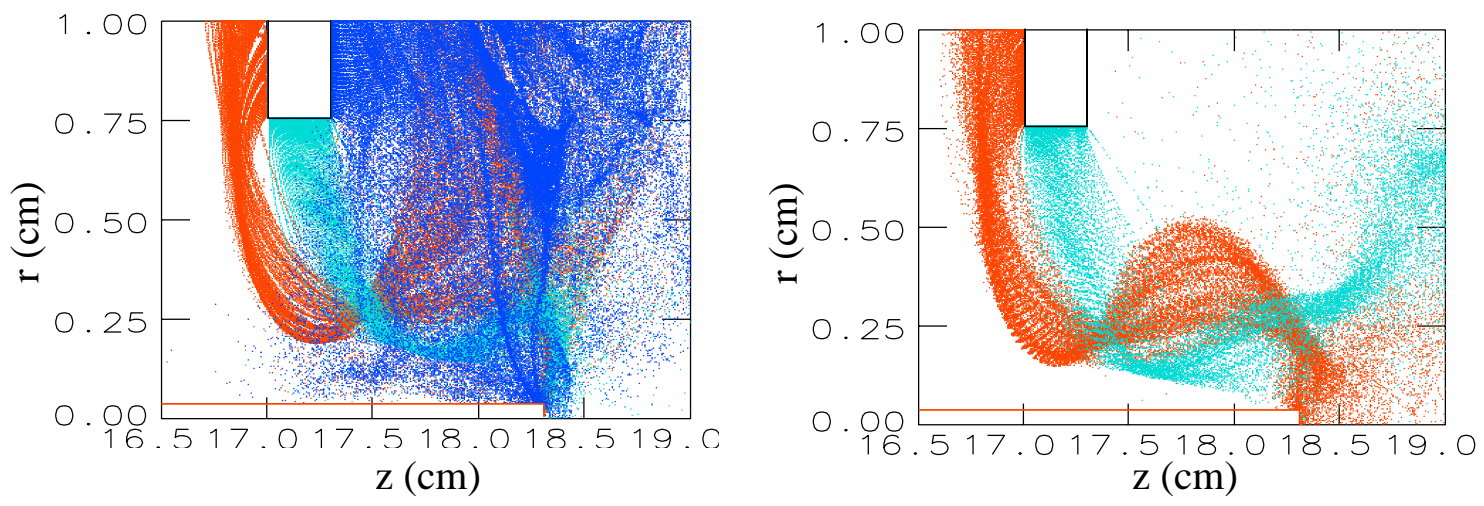

Figure 3: Comparison of the electron dynamics from simulations (at $t=14 \mathrm{ns)} \mathrm{for} r_{c} / r_{a}=20$ with (left panel) and without (right panel) the electron emission from downstream cathode surface. The run without the electron emission from downstream cathode surface shows an improved diode performance in which the electrons strike only the anode tip.

in Figure 3 for $r_{c} / r_{a}=20$. The left panel is a scatter plot of electrons from the first run that includes electron emission from all three cathode surfaces (electrons from the three cathode surfaces are plotted in different colors), whereas the right panel is a run in which the electron emission from the downstream cathode surface is suppressed by increasing the electric field threshold. It is apparent that the second run produces an electron source that strikes only the anode tip, whereas in the first run electrons appear in regions upstream of the tip along the anode shaft, which can adversely affect the diode performance.

\section{SUMMARY AND CONCLUSIONS}

We have analyzed, using 2-D PIC simulations, the electron physics in the rod-pinch diode using both the diode parameters used in the Cygnus experiment $r_{c} / r_{a}=12$ and large values of $r_{c} / r_{a}$. It is shown that in the case with $r_{c} / r_{a}=12$, the electron dynamics can be reasonably well approximated as a magnetized fluid. In contrast, simulations using large cathode-to-anode radius ratios, $r_{c} / r_{a}=$ 20 and 40, exhibit the presence of upstream-propagating electrons. An electron population emitted primarily from the downstream cathode surface has been identified as the source of the upstream-propagating electrons. These electrons can be described as unmagnetized and they propagate upstream in the presence of a downstream-directed electric field produced by the convection of the magnetized electrons that are moving towards the anode. Finally, a method is proposed for the suppression of the electron emission from the downstream cathode surface in order to reduce the upstream-propagating electrons and to improve the quality of the electron source. The proposed method is currently under experimental investigation. Direct comparison and validation of the electron physics discussed in this paper with experimental results will be discussed in a forthcoming paper.

\section{REFERENCES}

[1] J. R. Smith, R. Carlson, R. D. Fulton, et al., American Institute of Physics Conference Proceedings, $14^{\text {th }}$ International Conference on High-Power Particle Beams, Albuquerque, NM, USA, 2002, edited by T. A. Mehlhorn and M. A. Sweeney, No.650, p.135; J. R. Smith, R. Carlson, R. D. Fulton, et al., $29^{\text {th }}$ IEEE International Conference on Plasma Science, Banff, Alberta, Canada, 2002 (Los Alamos National Laboratory LA-UR-02-2969).

[2] R. A. Mahaffey, J. Golden, S. A. Goldstein, and G. Cooperstein, Appl. Phys. Lett. 33, 795 (1978).

[3] G. Cooperstein, R. J. Commisso, D. D. Hinshelwood, P. F. Ottinger, D. V. Rose, S. J. Stephanakis, S. B. Swanekamp, F. C. Young, Proceedings of the 12th International Conference on High-Power Particle Beams, Haifa, Israel, 1998, edited by M. Markovits and J. Shiloh (Institute of Electrical and Electronics Engineers, Piscataway, NJ, 1998), Vol.1, p.31.

[4] R. J. Commisso, G. Cooperstein, D. D. Hinshelwood, et al., IEEE Trans. Plasma Sci. 30, 338 (2002).

[5] F. C. Young, R. J. Commisso, R. J. Allen, et al., Phys. Plasmas 9, 4815 (2002).

[6] P. R. Menge, D. L. Johnson, J. E. Maenchen, D. C. Rovang, B. V. Oliver, D.V. Rose, and D. R. Welch, Rev. Sci. Instrum. 74, 3628 (2003).

[7] J. R. Smith, private communications (2003).

[8] G. Cooperstein, J. R. Boller, R. J. Commisso, D. D. Hinshelwood, D. Mosher, P. F. Ottinger, J. W. Schumer, S. J. Stephanakis, S. B. Swanekamp, B. V. Weber, and F. C. Young, Phys. Plasmas 8, 4618 (2001).

[9] T. Kwan, and C. Snell, "Methods of Monte Carlo Electron Transport in Particle-in-Cell Codes", Lecture Notes in Physics, in Monte Carlo Methods and Applications in Neutronics, Photonics, and Statistical Physics, edited by R. Alcouffe, R. Dautray, A. Forster, G. Ledanois, and B. Mercier (Springer, New York, 1985), p. 146.

[10] M. Berninger, T. Kwan, and L. Yin, Los Alamos National Laboratory Report LA-UR-040196 (2003). 